\title{
Prospects for Predicting Cycle 24
}

\author{
Arnab Rai Choudhuri \\ Department of Physics, Indian Institute of Science, Bangalore 560 012, India. \\ e-mail:arnab@physics.iisc.ernet.in
}

\begin{abstract}
Although we have reliable data of solar polar fields only from the mid-1970s, it seems that the polar field at a minimum is well correlated with the next cycle, but the strength of the cycle is not correlated with the polar field produced at its end. We explain this by suggesting that the Babcock-Leighton mechanism of poloidal field generation from tilted active regions involves randomness, whereas the other aspects of the dynamo process are more ordered. To model actual cycles, we have to 'correct' our theoretical dynamo model by 'feeding' information about the polar field at the minima. Following this process, we find that our model fits the observed sunspot numbers of cycles 21-23 reasonably well and predicts that cycle 24 will be the weakest in a century.
\end{abstract}

Key words. Sun: magnetic field-solar cycle-MHD.

\section{Background}

When we try to predict a solar cycle a few years ahead of time, have we reached the stage when we can regard our methodology to be slightly more reliable than betting in a horse race? That is the question we wish to discuss in this presentation.

Nearly three decades ago, it was suggested by Schatten et al. (1978) that the polar field at the preceding minimum gives an indication of the strength of the next solar cycle. If the polar field at a minimum is stronger than the usual, then the next cycle is likely to be strong, and vice versa. Let us discuss whether this method can be considered sufficiently reliable. We have systematic polar field data only from the mid1970s when WSO and MWO started regular observations of the polar field. Svalgaard et al. (2005) have computed a quantity DM, which is a proxy of the dipole moment of the Sun and which is a good measure of the polar field of the Sun at the minimum. Table 1 lists DM values at the end of cycles 20-23, along with the strengths of cycles 20-23. In Fig. 1, we plot the strengths of the cycle $n+1$ against the DM values at the end of cycles $n$. Although we have only 3 data points by now and one may justifiably question the statistical significance of any correlation based on 3 data points, all the 3 points lie so close to a straight line that it is difficult to believe that this is merely a statistical coincidence. If the solid straight line in Fig. 1 is assumed to be an approximate representation of a real correlation, then it is easy to predict the strength of the coming cycle 24 . Since DM value at the present minimum is about 120 (indicated by the dashed line), the predicted strength of cycle 24 can be merely read off from the vertical scale corresponding to the point where the dashed line cuts the solid line 
Table 1. Maximum strength of the cycle and the DM value at its end are listed against cycle number. The data for this table are taken from Svalgaard et al. (2005).

\begin{tabular}{ccc}
\hline $\begin{array}{c}\text { Cycle } \\
\text { number }\end{array}$ & $\begin{array}{c}\text { Maximum strength } \\
\text { of the cycle } R_{\max }\end{array}$ & $\begin{array}{c}\text { DM value at } \\
\text { the end of cycle }\end{array}$ \\
\hline 20 & 110.6 & 250 \\
21 & 164.5 & 245.1 \\
22 & 158.5 & 200.8 \\
23 & 120.8 & 119.3 \\
\hline
\end{tabular}

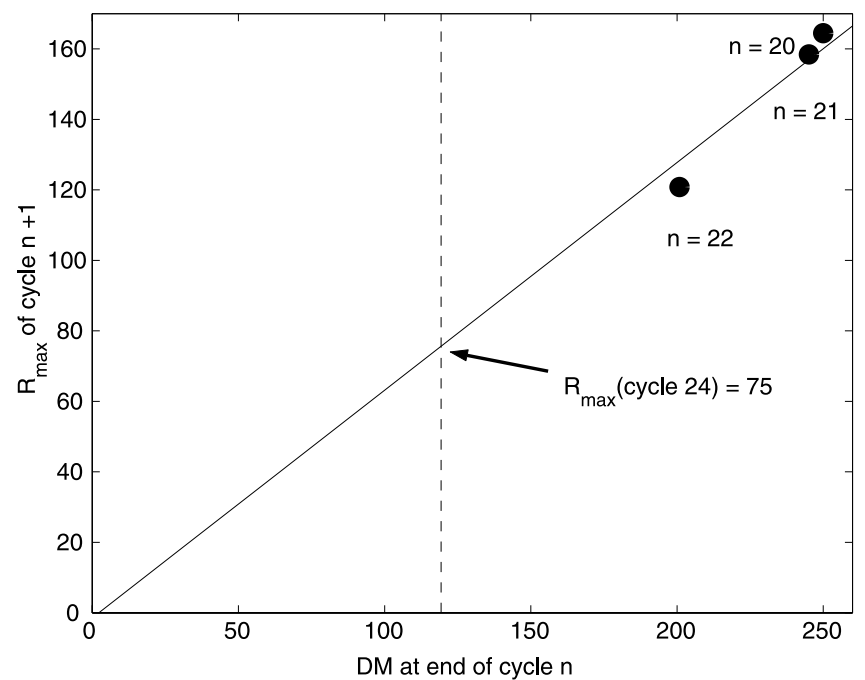

Figure 1. Strengths of solar cycles plotted against the DM values of polar fields at the preceding minima.

representing the correlation. This gives an $R_{\max }$ of about 75, suggesting that cycle 24 will be the weakest in a century. This is essentially the basis of the predictions made by Schatten (2005) and Svalgaard et al. (2005), who believe that cycle 24 will be very weak.

We now do the next interesting thing of plotting DM at the end of cycle $n$ against the strength of that cycle. This is shown in Fig. 2. It is clear even from the limited number of data points that there is no good correlation. This means that the strength of the cycle does not completely determine the polar field at the end of the cycle. A strong cycle is not necessarily followed by a strong polar field at its end. Figure 1 virtually suggests that the polar field at the end of a cycle in some way almost determines the next cycle. But we conclude from Fig. 2 that the strength of the cycle does not determine the polar field at its end in the same causal way. There is a break or at least a weakening in the chain of causality when we go from the maximum of a cycle to the following minimum. We actually need such a break in strict causality somewhere in the cycle because the solar cycles are irregular. If strict causality were followed everywhere, we would have expected the cycles to be much more regular. We shall further discuss the implications of these figures later in our presentation. 


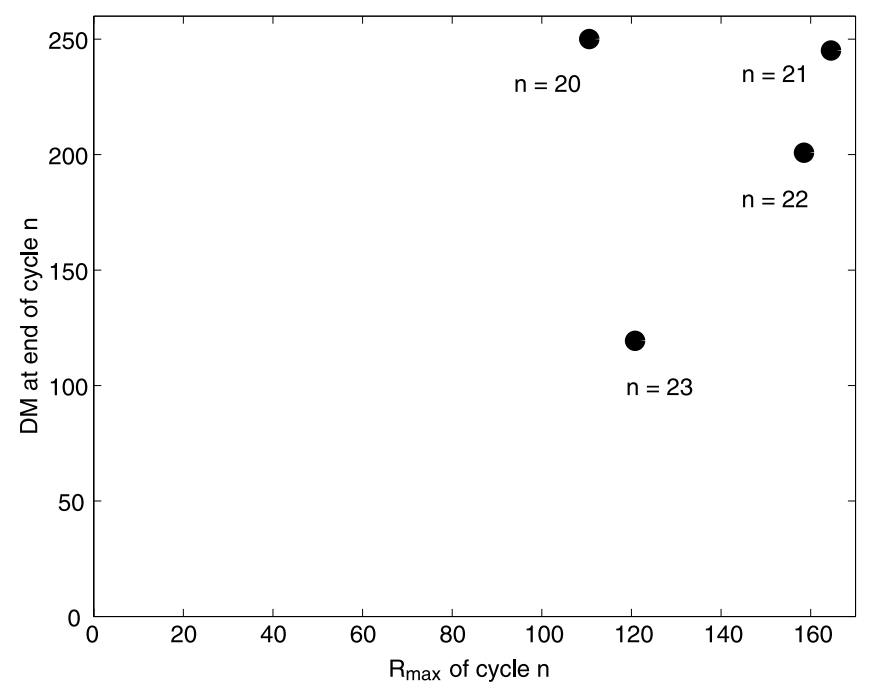

Figure 2. DM values of polar fields at the minima plotted against the strengths of the previous solar cycles.

Since the solar cycle is believed to be produced by a hydromagnetic dynamo process, we would now like to raise the question whether the solar dynamo theory can be applied to predict solar cycles and whether the empirical method of using the polar field strength at the preceding minimum as an indicator for the next cycle can be justified theoretically from dynamo models. Only within the last few years, solar dynamo models have become sufficiently sophisticated and realistic to address these issues. Dikpati \& Gilman (2006) have published the first prediction of cycle 24 based on a solar dynamo model. They claim that cycle 24 will be the strongest in 50 years. If their prediction turns out to be true, then the fourth data point in Fig. 1 will lie on the dashed line a little above the upper boundary of the figure, far away from the solid line which indicates a possible correlation! Several dynamo theorists have been harshly critical of the work of Dikpati \& Gilman (2006). Tobias et al. (2006) make the following comment on this work: "Any predictions made with such models should be treated with extreme caution (or perhaps disregarded), as they lack solid physical underpinnings." While we are also highly critical of the Dikpati-Gilman work for reasons which we shall explain in section 3, we cannot also accept the opposite extreme viewpoint of Tobias et al. (2006), who suggest that the solar dynamo is a nonlinear chaotic system and predictions are impossible or useless. If that is the case, then we are left with no explanation for the correlation seen in Fig. 1.

This state of affairs made us feel that it was important to take a fresh look at the problem from a new angle. Our first results on this subject (Choudhuri et al. 2007) are discussed in section 2 . Finally section 3 presents our concluding remarks, contrasting our methodology with that of Dikpati \& Gilman (2006).

\section{Our model}

Some processes in nature can be predicted and some cannot. We can easily calculate the trajectory of a projectile by using elementary mechanics. On the other hand, when 
a dice is thrown, we cannot predict which side of the dice will face upward when it falls. Is the solar dynamo more like the trajectory of a projectile or more like the throw of a dice? Our point of view is that the solar dynamo is not a simple unified process, but a complex combination of several processes, some of which are predictable and others not. Let us look at the processes which make up the solar dynamo.

Most of the current solar dynamo models combine three basic processes:

(i) The strong toroidal field is produced by the stretching of the poloidal field by differential rotation in the tachocline.

(ii) The toroidal field generated in the tachocline gives rise to active regions due to magnetic buoyancy and then the decay of tilted bipolar active regions produces the poloidal field by the Babcock-Leighton mechanism.

(iii) The meridional circulation advects the poloidal field first to high latitudes and then down to the tachocline.

Two-dimensional mean field dynamo models based on these three processes were first constructed about a decade ago (Choudhuri et al. 1995; Durney 1995). We believe that the processes (i) and (iii) are reasonably ordered and deterministic. In contrast, the process (ii) involves an element of randomness due to the following reason. The poloidal field produced from the decay of a tilted bipolar region by the BabcockLeighton process depends on the tilt. While the average tilt of bipolar regions at a certain latitude is given by Joy's law, we observationally find quite a large scatter around this average. Presumably the action of the Coriolis force on the rising flux tubes gives rise to Joy's law (D'Silva \& Choudhuri 1993), whereas convective buffeting of the rising flux tubes in the upper layers of the convection zone causes the scatter of the tilt angles (Longcope \& Choudhuri 2002). This scatter in the tilt angles certainly introduces a randomness in the poloidal field generation process.

The poloidal field gets built up during the declining phase of the cycle and becomes concentrated near the poles during the minimum. The polar field at the solar minimum produced in a theoretical mean field dynamo model is some kind of 'average' polar field during a typical solar minimum. The observed polar field during a particular solar minimum may be stronger or weaker than this average field. The theoretical dynamo model has to be updated by feeding the information of the observed polar field in an appropriate way, in order to model particular cycles. We propose the following methodology for this in our recent paper (Choudhuri et al. 2007). We run the dynamo code from a minimum to the next minimum in the usual way. If we want to model a particular minimum with a known observed value of DM, then we multiply the poloidal field of the theoretical model stopped at the minimum by a constant factor everywhere above $0.8 \mathrm{R}_{\odot}$ such that its amplitude becomes proportional to the value of DM. Since some of the poloidal field at the bottom of the convection zone may have been produced in the still earlier cycles, we leave it unchanged by not doing any updating below $0.8 \mathrm{R}_{\odot}$. Only the poloidal field produced in the last cycle which is concentrated in the upper layers gets updated to make it correspond to the observed DM. After this updating, we run the code till the next minimum, when the code is again stopped and the same procedure is repeated by using the value of DM at that minimum. Our solutions are now no longer self-generated solutions from a theoretical model alone, but are solutions in which the random aspect of the dynamo process has been corrected by feeding the observational data of polar fields into the theoretical model. 

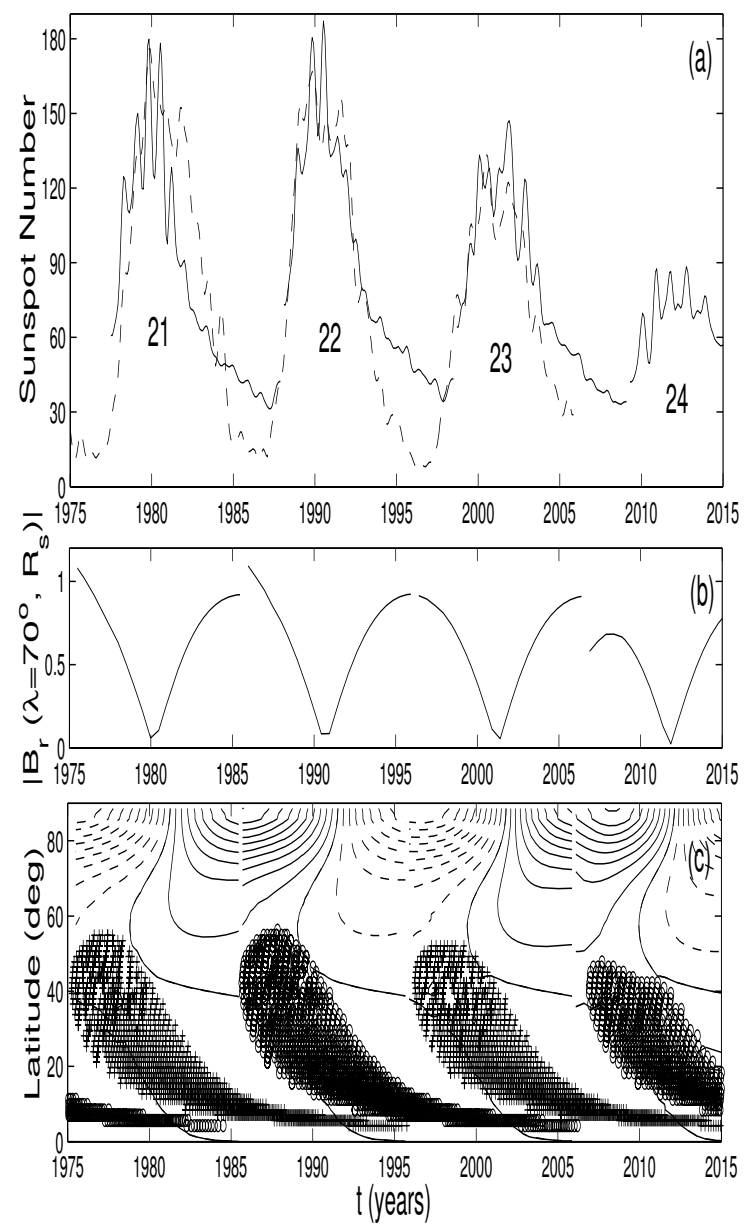

Figure 3. Theoretical results from our dynamo model for cycles 21-24. (a) The theoretical monthly smoothed sunspot numbers (solid line) superposed on the monthly smoothed sunspot numbers from observation (dashed line). (b) A plot of $B_{r}$ at the surface at a latitude of $70^{\circ}$. (c) The theoretical butterfly diagram, with contours of $B_{r}$ at the surface in the time-latitude plot. This figure is taken from Choudhuri et al. (2007).

Our calculations are based on a dynamo model presented in some of the earlier papers from our group (Nandy \& Choudhuri 2002; Chatterjee et al. 2004). Since values of DM are available only from the minimum at the end of cycle 20 and afterwards, Choudhuri et al. (2007) could apply their methodology to model the last few cycles only. Figure 3 shows their results. Since the value of DM at the end of cycle 23 (i.e., at the present time) is available, calculations could be continued till the minimum at the end of cycle 24. The top panel in Fig. 3 shows theoretical sunspot numbers (solid line) superposed on the observed sunspot numbers (dashed line). We find that cycles 21-23 are modeled reasonably well by our solar dynamo model. Cycle 24 comes out much weaker than even the reasonably weak cycle 23 , suggesting that it will probably be the weakest cycle in a century. The middle panel plots the radial magnetic field at a point on the surface. We see the discontinuities at the minima when the poloidal field is 
updated in accordance with observational data. The bottom panel gives the theoretical butterfly diagram.

The result that cycle 24 will be a weak cycle seems like a very robust result from our dynamo model and does not change significantly on varying various parameters within reasonable limits. It may be noted that the strength of cycle 24 predicted by us would make it lie exactly on the straight line in Fig. 1 indicating the possible correlation.

\section{Conclusion}

Choudhuri (1992) postulated several years ago that stochastic fluctuations in the poloidal field generation mechanism may be the cause of irregularities in the solar cycle. We now identify the randomness in the Babcock-Leighton process as the source of these fluctuations. Because of this randomness, a strong cycle is not necessarily followed by a strong polar field at its end and vice versa. The lack of correlation seen in Fig. 2 can be taken as a confirmation of our hypothesis that the Babcock-Leighton process involves randomness. Although we have reliable polar field data only for the last 4 minima, the polar field at earlier times can be indirectly estimated from polar faculae data (Sheeley 1991) or positions of filaments (Makarov et al. 2001). These data also suggest that the strength of the polar field at a minimum determines the strength of the next cycle, but the strength of the cycle is not very well correlated with the polar field produced at its end.

Theoretical considerations alone do not allow us to build a model of actual solar cycles. We have to feed some relevant information from observational data into our theoretical model. Since the polar field produced at the end of a cycle cannot be predicted correctly from a mean field model (due to the randomness in the production process), we suggest that 'correcting' the polar field at the minima by using observational data is the best way of feeding relevant observational information into the theoretical model. In contrast, Dikpati \& Gilman (2006) use the sunspot area data as the source of poloidal field in their model. In view of the randomness in the BabcockLeighton process leading to a lack of correlation between the sunspots of a cycle and the polar field at its end (as seen in Fig. 2), we are highly doubtful whether the methodology of Dikpati \& Gilman (2006) is correct. We shall have a verdict from the Sun-god himself within the next 4-5 years whether their prediction or our prediction comes closer to the truth.

The rising phase of a solar cycle is dominated by processes like the advection of the poloidal field by meridional circulation and its stretching by the differential rotation in the tachocline, which are ordered and deterministic processes. So we suggest that the rising phase of a cycle is reasonably deterministic, allowing us to predict the strength of a cycle a few years ahead of time by using the polar field data. On the other hand, the declining phase of a solar cycle is not predictable, since it is dominated by the Babcock-Leighton process which involves randomness. Hence, it may never be possible to predict the strength of a maximum more than 7-8 years ahead of time even when we have better dynamo models and more high-quality magnetic field data.

\section{Acknowledgements}

The work presented here would not have been possible without the dedicated efforts of my collaborators, Piyali Chatterjee and Jie Jiang. I also thank Axel Brandenburg, 
Paul Charbonneau, Dibyendu Nandy, Leif Svalgaard and Jingxiu Wang for valuable discussions.

\section{References}

Chatterjee, P., Nandy, D., Choudhuri, A. R. 2004, Astron. Astrophys., 427, 1019.

Choudhuri, A. R. 1992, Astron. Astrophys., 253, 277.

Choudhuri, A. R., Chatterjee, P., Jiang, J. 2007, Phys. Rev. Lett., 98, 131103.

Choudhuri, A. R., Schüssler, M., Dikpati, M. 1995, Astron. Astrophys., 303, L29.

D’Silva, S., Choudhuri, A. R. 1993, Astron. Astrophys., 272, 621.

Dikpati, M., Gilman, P. A. 2006, Astrophys. J., 649, 498.

Durney, B. R. 1995, Solar Phys., 160, 213.

Longcope, D., Choudhuri, A. R. 2002, Solar Phys., 205, 63.

Makarov, V. I., Tlatov, A. G., Callebaut, D. K., Obridko, V. N., Shelting, B. D. 2001, Solar Phys., 198, 409.

Nandy, D., Choudhuri, A. R. 2002, Science, 296, 1671.

Schatten, K. H. 2005, Geophys. Res. Lett., 32, L21106.

Schatten, K. H., Scherrer, P. H., Svalgaard, L., Wilcox, J. M. 1978, Geophys. Res. Lett., 5, 411.

Sheeley, N. R. 1991, Astrophys. J., 374, 386.

Svalgaard, L., Cliver, E. W., Kamide, Y. 2005, Geophys. Res. Lett., 32, L01104.

Tobias, S., Hughes, D., Weiss, N. 2006, Nature, 442, 26. 